\title{
A Correlative Study of N-Cadherin Expression with Different Grades of Oral Squamous Cell Carcinoma Projecting as a Marker of Epithelial to Mesenchymal Transition in Tumor Progression
}

\author{
Harishchandra Rai K ${ }^{1 *}$, Junaid Ahmed ${ }^{2}$
}

\begin{abstract}
Background: Epithelial cells typically express E-cadherin where as N-cadherin expressed by mesenchymal cells. The epithelial to mesenchymal transition (EMT) is a process by which epithelial cells lose their cell polarity and cell-cell adhesion, and gain migratory and invasive properties to become mesenchymal cells. EMT is typical for carcinoma cells during tumor progression and correlate with the local invasiveness and metastatic potential of the tumor. Oral squamous cell carcinoma is a malignant neoplasm arising from the mucosal epithelium of the oral cavity. It can be classified as well; moderate and poor depends on a tumor cells resemblance to its tissue of origin. Materials and Methods: A total of 130 cases of histopathologically diagnosed as OSCC were selected for the study, out of which 66,38 and 26 were well, moderate and poorly differentiated respectively. One section was stained with Haematoxylin and Eosin and the other section for $\mathrm{N}$-cadherin immunohistochemical study. Then the N-cadherin expression was correlated histopathologically with different grades of OSCC. Statistical analysis was carried out mainly by Chi-Square analysis. Results: Among the 66 cases of WDSCC mean value of N-cadherin expression was 1.79, 38 cases of MDSCC mean value of $\mathrm{N}$-cadherin expression was 4.16 and among the 26 cases of PDSCC the mean value was 6.38 .That means the value of $\mathrm{N}$ - cadherin expression was progressively increasing with decreased differentiation of the tumor cells. The statistical analysis also shown it was highly significant $(\mathrm{P}<0.001)$. Conclusion: A correlative study of $\mathrm{N}$-cadherin expression with different grades of OSCC will be useful to predict the state of tumor progression and also it may give accuracy for histopathogical grading of the tumor.
\end{abstract}

Keywords: Oral squamous cell carcinoma- epithelial-mesenchymal transition- N-cadherin

Asian Pac J Cancer Prev, 20 (8), 2327-2332

\section{Introduction}

Epithelial cell to cell junctions provide tissue integrity and promote cellular polarity. The adherens junction plays a pivotal role in regulating the activity of the entire junctional complex, and the major adhesion molecules in the adherens junctions are the cadherins (Masato Maeda et al., 2004). the cadherins comprises a family of $\mathrm{Ca}^{2+}$-dependant adhesion molecules that function to mediate the cell-to-cell binding that is critical to the maintenance of tissue structure and morphogenesis. And also cadherin adhesion plays a signaling role during cell growth and differentiation (Sung et al., 2007).

Cadherins are single pass trasmembrane proteins that are synthesized with a single peptide (SP) and pro region (pro), which are removed during protein processing. The extracellular domain comprises five homologous repeats
(EC1-EC5) that are bridged by calcium ions $\left(\mathrm{Ca}^{2+}\right)$.The cytoplasmic domain binds to p120-catenin (p120ctn) near the plasma membrane and to $\beta$-catenin near the $\mathrm{C}$-terminus. B-catenin binds to $\alpha$-catenin to link the cadherin complex to the actin cytoskeleton (Margaret et al., 2007).

N-cadherin was first identified in 1982 by Grunwald and others as a $130 \mathrm{kD}$ molecule in the chick neural retina that was protected by calcium from proteolysis, and in 1984 A-CAM was identified (now called N-cadherin) as a molecule that was localized at the adherens junctions .In the nomenclature of $\mathrm{CD}$ antigens the new designation for N-cadherin is CD325, N-cadherin is also known as neural-cadherin, non-epthelial cadherin or cadherin-2. (Lara et al., 2004).

The epithelial-mesenchymal transition or transformation (EMT) is a process by which epithelial 
cells lose their cell polarity and cell-cell adhesion, and gain migratory and invasive properties to become mesenchymal cells (Derek, 2005). EMT is considered as a reversible biologic process that is important for normal embryogenesis and organ development from single-layered to multilayered organisms, particularly during gastrulation and neural crest cell migration. One of the best-studied and -known processes of EMT is the breakdown of intercellular adhesion between epithelial cells by inhibiting expression of E-cadherin (epithelial cadherin); whereas the cells that undergo the EMT process aberrantly express higher levels of $\mathrm{N}$-cadherin (mesenchymal cadherin). This process is called "cadherin switching" (Suttichai and Anak, 2012).

EMT is a natural process that can be found in several pathophysiological conditions in human adults. Particularly, EMT is a necessary component of the inflammatory process and normal wound healing in adulthood. Loss of epithelial morphology and acquisition of mesenchymal characteristics (EMT), are typical for carcinoma cells during tumor progression and correlate with the local invasiveness and metastatic potential of the tumor (Suttichai et al., 2012; Silvana et al., 2012; Michael et al., 2009).

Oral squamous cell carcinoma (OSCC) is a malignant neoplasm arising from the mucosal epithelium of the oral cavity (Ankur et al., 2010). A tumor that is mature enough to closely resemble its tissue of origin are well-differentiated squamous cell carcinoma. In contrast, a tumor with much cellular and nuclear pleomorphism and with little or no keratin production may be so immature that it becomes difficult to identify the tissue of origin are poorly differentiated or anaplastic. A tumor with a microscopic appearance somewhere between these two extremes is labeled a "moderately differentiated" carcinoma (Brad et al., 2008; Rajendran et al., 2012).

However there are so many studies on N-cadherin expression with many malignant tumors but the $\mathrm{N}$-cadherin study on OSCC was very limited. So we are here to study and compare the N-cadherin expression in OSCC with different histopathological grades relevant to tumor progression and prognosis.

\section{Materials and Methods}

Formalin fixed, paraffin embedded block specimen of both incisional and excisional biopsies of oral squamous cell carcinoma(OSCC) were obtained from the archives of Department of Oral Pathology and Microbiology, K.V.G Dental College and Hospital, Sullia and Kidwai Memorial Institute of Oncology, Bangalore.The slides were graded according to Modified Broders's system as well, moderate and poorly differentiated squamous cell carcinoma respectively. Among the total of 130 cases, $66(50.8 \%)$ cases of well differentiated squamous cell carcinoma(WDSCC), 38 (29.2\%) cases of moderately differentiated squamous cell carcinoma(MDSCC) and $26(20.0 \%)$ cases of poorly differentiated squamous cell carcinoma(PDSCC) were selected. The clinical details of all the cases like patients age, sex, site of the lesion and habits and its duration were systematically tabulated. From each selected cases two serial sections of 5 microns were prepared by using microtome. One section was stained with Haematoxylin and Eosin and the other section for $\mathrm{N}$-cadherin immunohistochemical study. All the slides were methodically evaluated by a single blinded observer in order to eliminate inter-observers bias.

Immunohistochemistry for $\mathrm{N}$-cadherin expression were carried out by using a monoclonal antibody and polymer Horseradish Peroxidase Detection System (BioGenex, CA, USA). Standardization and monitoring of the procedure of immunostaining was done by an expert from BioGenex Life Systems Pvt. Ltd.The normal oral epithelium which shows no expression of $\mathrm{N}$-cadherin has been taken as a negative control. N-cadherin is found to be strongly expressed in breast cancer used as a positive control.

Formalin fixed paraffin embedded wax blocks were used to obtain $5 \mu \mathrm{m}$ sections for immunostaining. The cut sections were then transferred on to poly-L-lysine coated slides. Incubation was done at a temperature range of 37-40 degrees celsius overnight. After deparafinization,antigen retrieval was carried out by heat treatment. Endogenous peroxidase activity was quenched by treating the sections with 3\% hydrogen peroxide for 6 minutes. The slides were then thoroughly rinsed in Tris buffer saline (pH-7.6) and then blotted. The slides were treated with Background Punisher for 10 minutes to prevent nonspecific binding of secondary antibody. Slides were then rinsed with Tris buffer saline and blotted gently.

The sections were incubated with monoclonal rabbit anti-human N-cadherin antibody for one hour at room temperature. The procedure was carried out in a moist chamber and the sections were then rinsed in Tris wash buffer ( 2 changes) for 5 minutes each. The slides were incubated with MACH $4 \mathrm{HRP}$ polymer for 20 minutes. The solution comprises of secondary antibody conjugated to non-biotinylated polymer HRP reagent. Again slides were rinsed in wash buffer ( 2 changes) for 5 minutes each and blotted gently. The substrate solution consists of mixture of (3, 3'-diaminobenzidine tetrahydrochloride) DAB buffer $(1 \mathrm{ml})$ and DAB chromogen (1 drop) to be added and the slides are rinsed with distilled water for 5 minutes. The slides were counterstained with Harris haematoxylin and mounted with DPX.

The slides were viewed in a bright field microscope at a magnification of 200x and $400 \mathrm{x}$ with objective used are 20x and 40x respectively in 10 different fields to analyze the percentage of cells showing positivity and the color intensity as well. The color intensity and percentage scores were multiplied to give a composite score of 1-9 for each case. The final staining score was defined as follows: 0 : negative, 1-3: weak, 4-6: moderate and 7-9: strong. All the statistical analysis was carried out by using the SPSS version 20 software.

\section{Results}

$\mathrm{N}$-cadherin positive staining was mainly observed with cell membrane and also in part of adjacent cytoplasm. 
DOI:10.31557/APJCP.2019.20.8.2327

$\mathrm{N}$-Cadherin for Grading Oral Squamous Cell Carcinoma

Table 1. A Comparative Analysis of Total N-Cadherin Expression Score with Different Grades of OSCC

\begin{tabular}{llcccccccc}
\hline OSCC & & \multicolumn{7}{c}{ Total Score } & \multicolumn{3}{c}{ Total } \\
& & 0 & 1 & 2 & 3 & 4 & 6 & 9 & 06 \\
\hline WDSCC & Count & 9 & 25 & 19 & 3 & 7 & 3 & 0 & 66 \\
& $\%$ & $100 \%$ & $96.20 \%$ & $86.40 \%$ & $25.00 \%$ & $23.30 \%$ & $14.30 \%$ & $0.00 \%$ & $50.80 \%$ \\
\multirow{2}{*}{ MDSCC } & Count & 0 & 1 & 3 & 5 & 19 & 10 & 0 & 38 \\
& $\%$ & $0.00 \%$ & $3.80 \%$ & $13.60 \%$ & $41.70 \%$ & $63.30 \%$ & $47.60 \%$ & $0.00 \%$ & $29.20 \%$ \\
\multirow{2}{*}{ PDSCC } & Count & 0 & 0 & 0 & 4 & 4 & 8 & 10 & 26 \\
& $\%$ & $0.00 \%$ & $0.00 \%$ & $0.00 \%$ & $33.30 \%$ & $13.30 \%$ & $38.10 \%$ & $100.00 \%$ & $20.00 \%$ \\
\multirow{2}{*}{ Total } & Count & 9 & 26 & 22 & 12 & 30 & 21 & 10 & 130 \\
& $\%$ & $100.00 \%$ & $100.00 \%$ & $100.00 \%$ & $100.00 \%$ & $100.00 \%$ & $100.00 \%$ & $100.00 \%$ & $100.00 \%$ \\
\hline
\end{tabular}

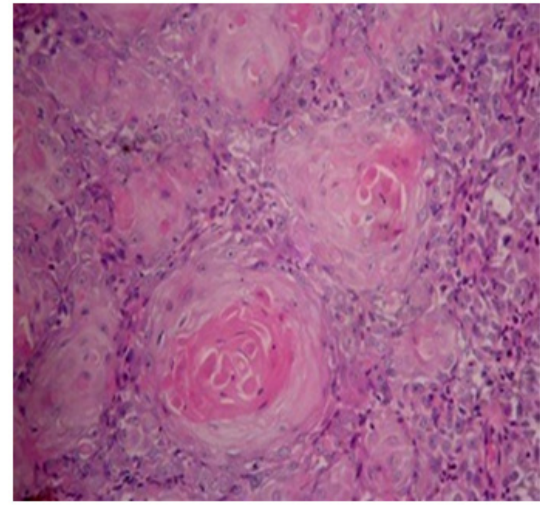

Figure 1. WDSCC H\&E Stain (400X)

Among the total of 66 cases of WDSCC 9 (100\%), 25 (96.2\%), 19 (86.4\%), 3 (25\%), 7 (23.3\%) and $3(14.3 \%)$ cases were scored totally $0,1,2,3,4$ and 6 respectively (Table 1). But there was no cases have been scored 9 but the majority of $96.2 \%$ cases were scored minimum of 1. Out of 38 cases of MDSCC 1 (3.8\%), $3(13.6 \%), 5$ (41.7\%), 19 (63.3\%) and 10 (47.6\%) cases were scored totally 1, 2, 3, 4 and 6 respectively. But there was no minimum of 0 score and maximum of 9 score in MDSCC but the majority of $63.3 \%$ cases were scored moderate of 4. Among the total of 26 cases of PDSCC 4 (33.3\%), $4(13.3 \%), 8(38.1 \%)$ and $10(100 \%)$ cases were scored $3,4,6$ and 9 respectively.And majority of cases scored maximum of 9 . Among the 130 cases, 9 cases have shown no staining or zero score and all these cases were WDSCC, 10 cases have shown maximum staining of score 9 and all these cases were PDSCC. Chi-square analysis of this

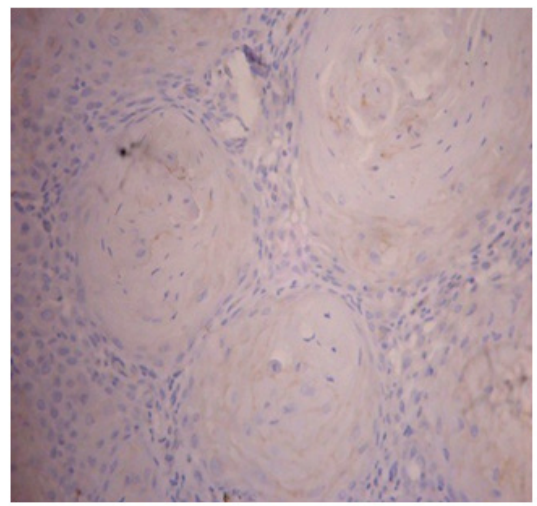

Figure 2. Week Expression of N-Cadherin in WDSCC $(400 \mathrm{x})$

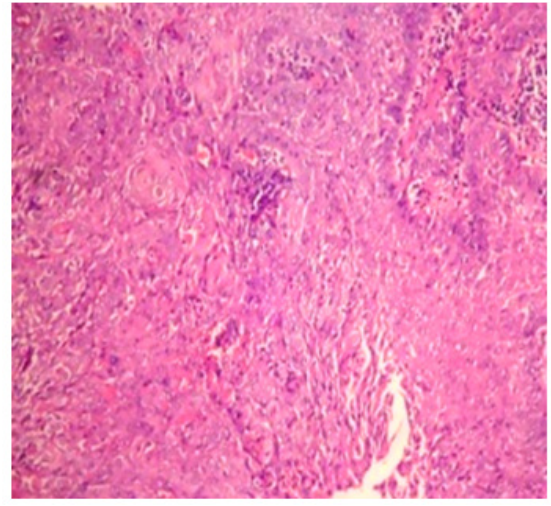

Figure 3. MDSCC H\&E Stain (200X)

comparison between Total $\mathrm{N}$-cadherin score with different grades of OSCC was found to be statistically very highly significant $(\mathrm{P}=<0.001)$.

Mean value of N-cadherin for WDSCC, MDSCC and PDSCC were 1.79, 4.16 and 6.38 respectively, so it is increasing with decreased differentiation of the tumor cells. The statistical analysis of the mean $\mathrm{N}$ - cadherin

Table 2. Mean N-Cadherin Expression Value for OSCC

\begin{tabular}{lccccc}
\hline OSCC & $\mathrm{N}$ & Mean & STD. Deviation & $\mathrm{F}$ & $P$ \\
\hline WDSCC & 66 & 1.79 & 1.45195 & & \\
MDSCC & 38 & 4.16 & 1.32596 & & \\
PDSCC & 26 & 6.38 & 2.35078 & 79.31 & $<0.001$ \\
\hline
\end{tabular}

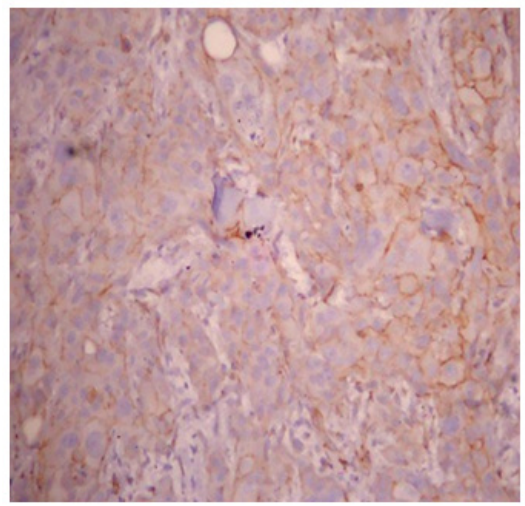

Figure 4. Moderate Expression of N-Cadherin in $\operatorname{MDSCC}(200 \mathrm{X})$

Asian Pacific Journal of Cancer Prevention, Vol 20 


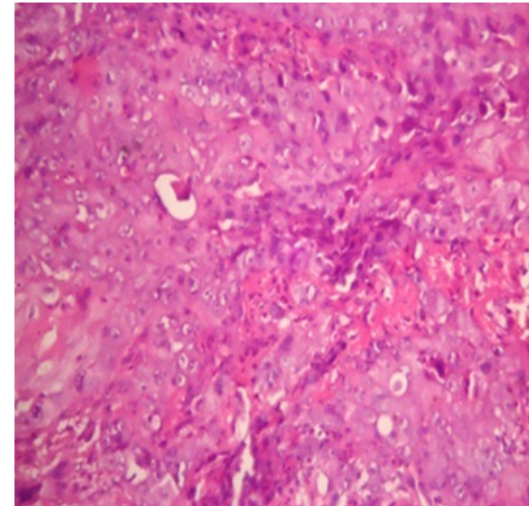

Figure 5. PDSCC H and E Stain (200X)

value for OSCC was very highly significant $(\mathrm{P}=<0.001)$ (Table 2).

\section{Discussion}

Oral squamous cell carcinoma is one of the most prevalent tumors of the head and neck region of epithelial origin. Loss of epithelial morphology and acquisition of mesenchymal characteristics termed as EMT are typical for carcinoma cells during tumor progression and correlate with local invasiveness and metastatic potential of the tumor. As in the oral squamous cell carcinoma EMT resulting in tumor cells become less differentiating from its original cells, so accordingly we will grade histopathologically well, moderate and poorly differentiated squamos cell carcinoma. The loss of differentiation from the original cells means tumour cells are gaining mesenchymal phenotype, and this will be better indicated by mesenchymal marker such as N-cadherin.

$\mathrm{N}$-cadherin study on OSCC are very limited, although M Di Domenico et al study showed the absence of $\mathrm{N}$-Cadherin staining in superficial well-differentiated cancer cells and strong staining in deeply infiltrating cancer cells. Finally, they found a statistically significant correlation between N-Cadherin expression and some histopathological parameters including grade and a statistical trend for stage. In our study also $\mathrm{N}$ - cadherin expression was increasing with high grade of OSCC. So the WDSCC have shown lower score of activity whereas PDSCC had higher score for N- cadherin activity.

Broder's histopathological classification on SCC is mainly based on the proportion of the neoplasm resembling normal squamous epithelium. Accordingly, tumors were graded on the basis of degree of differentiation and keratinization of tumor cells, so it is classified as well, moderate and poor. So WDSCC was diagnosed because its resemblance to cells of its origin, i.e. oral epithelium, so still the tumor cells not transformed into mesenchymal phenotype, and $\mathrm{N}$-cadherin expression either absent or less. PDSCC was diagnosed because of its lack of differentiation to its origin cells and that means the tumor cells gaining mesennchymal phenotype and so the cells were expressing high activity of N-cadherin. MDSCC were expressed with moderate activity of $\mathrm{N}$-cadherin. So

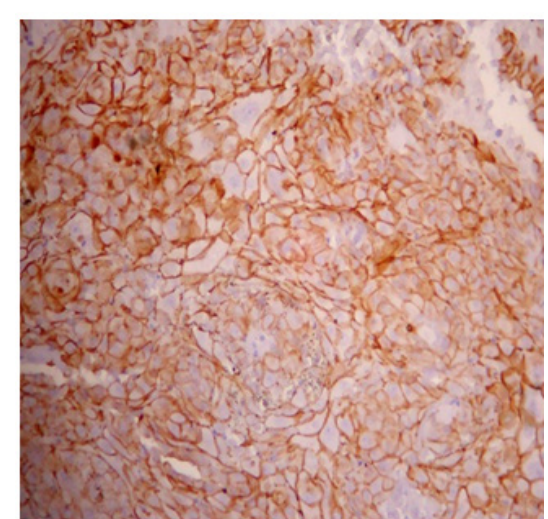

Figure 6. Strong expression of N-Cadherin in PDSCC (200X)

the $\mathrm{N}$-cadherin expression in our study was increasing with decreased differentiation. The statistical analysis were also confirmed with high significant value $(\mathrm{P}=<0.001)$.

M DI Domenico et al also found that N-Cadherin expression were characterized by a malignant phenotype associated with a worse clinical outcome, reduced overall survival and a major tendency for loco-regional invasion and metastasis. This study underlines the central role of $\mathrm{N}$-Cadherin in oral carcinogenesis, underlining its function as a potential therapeutic target and its potential value as a prognostic tool in the clinical management of OSCC (Domenico et al., 2011).

The N-cadherin positive cells in more than $60 \%$ and maximum staining intensity was observed majority in PDSCC. And minimum of less than $30 \%$ positive cells and minimum or no staining was seen majority in WDSCC. Among the 66 cases of WDSCC mean value of N-cadherin expression was $1.79,38$ cases of MDSCC mean value of $\mathrm{N}$-cadherin expression was 4.16 and among the 26 cases of PDSCC the mean value was 6.38.That means the value of $\mathrm{N}$ - cadherin expression was progressively increasing with decreased differentiation of the tumor cells. The statistical analysis also shown it was highly significant $(\mathrm{P}<0.001)$.

Snug et al., (2007) had the conclusion that reduced E-cadharin and positive $\mathrm{N}$-cadharin expression are closely associated in OSCC, suggest that cadherin switching probably plays an a important role in the development of OSCC and metastasis. Nguyen et al., (2011) were also suggested that cadherin switching might be considered as a discrete critical event in EMT and metastatic potential of HNSCC. Kathryn et al., (2006) also observed the increased invasiveness seen in $\mathrm{N}$-cadherin expressing cells of the OSCC.

The expression of TGF- $\beta 1, \mathrm{~N}$-cadherin, TP53 and $\mathrm{RB} 1 \mathrm{CC} 1$ increased in different stages of OSCC in both humans and mice. The expression of E-cadherin decreased from normal oral mucosa to OSCC, and increased in lymph node metastases in both human and mouse samples. (Mengzhu et al., 2017). OSCC cells in tumor nests with a cut-off point of 70 years old was the best fit model. These results suggest that $\mathrm{N}$-cadherin has significant value in prognostic predictions for OSCC patients (Yuu et al., 2017).

Other than OSCC, Ke et al., (2009) were observed the decreased E-cadherin expression and increased 
$\mathrm{N}$-cadherin expression more frequently in advanced Esophageal squamous cell carcinoma (ESCC) than in low grade ESCC,confirming that the down-regulation of E-cadherin expression and up-regulation of N-cadherin expression were closely associated with the infiltration, invasion and metastasis of ESCC. Erika et al., (2004) were found the $\mathrm{N}$-cadherin expression correlated with neural invasion, histological type, fibroblast growth factor expression in primary tumors of the pancreatic carcinoma.

Nhan et al., (1999) study on prostate cancer suggested that, $\mathrm{N}$-cadherin mediates a less stable cell-cell adhesion and may allow for carcinoma cell invasion and stromal interactions. Marvin et al., (1999) study on breast carcinoma showed that $\mathrm{N}$-cadherin promotes motility and invasion and that decreased expression of E-cadherin does not necessarily correlate with motility or invasion in breast cancer cells. Nakashima et al., (2003) and others study on lung cancer revealed the frequency of hypervascular tumours was significantly higher for $\mathrm{N}$-cadherin-positive carcinomas than for $\mathrm{N}$-cadherin-negative carcinomas. Whereas the 5-year survival rate of patients with $\mathrm{N}$-cadherin-positive tumours was significantly lower than that of patients with N-cadherin-negative tumours.

So the results of our study suggested that $\mathrm{N}$-cadherin expression in OSCC can be considered as an accurate marker of EMT. As the tumor progresses from WDSCC to PDSCC, the N-cadherin expression was also progressively increasing. $\mathrm{N}$-cadherin is the mesenchymal marker so obviously it is absent in epithelial cells but positive for mesenchymal cells. The histopathological grading of OSCC was based on its resemblance to cells of its origin, i.e. epithelial cells. Among the cases of WDSCC $\mathrm{N}$-cadherin expression was absent or minimum but in PDSCC this activity was maximum and in MDSCC it was moderate, it means that $\mathrm{N}$-cadherin can be considered as tumor marker of EMT in tumor progression from early invasion through different grades of OSCC to metastasis.

In conclusion, $\mathrm{N}$-cadherin is the mesenchymal cadherins and absent in epithelial cells, expression in tumor cells of oral squamous cell carcinoma indicates epithelial to Mesenchymal transition. A correlative study of $\mathrm{N}$-cadherin expression with different grades of oral squamous cell carcinoma will be useful to predict the state of tumor progression and also it may give accuracy for histopathogical grading of the tumor.

\section{Acknowledgments}

This study was supported by the pacific academy of higher education and research university Udaipur, Rajasthan and provided technical support by the KVG dental college and hospital Sullia, Karnataka. All the procedures of these in vitro studies were in accordance with ethical standards of both the institutional research committee.

\section{References}

Ankur B, Sonal S, Monali C (2010). Histopathological grading systems in oral squamous cell carcinoma: A review. J Int Oral Health, 2, 1-10.
Brad WN, Douglas DD, Carl MA, et al(2008). Oral and maxillofacial pathology. 3rd Edition, pp 409-21.

Derek CR (2005). Epithelial-mesenchymal transition. J Cell Sci, 118, 4325-6.

Domenico MD, Pierantoni GM, Feola A, et al (2011). Prognostic significance of N-Cadherin expression in oral squamous cell carcinoma. Anticancer Res, 31, 4211-18.

Doshi NP, Shah SA, Patel KB, et al (2011). Histological grading of oral cancer: A comparison of different systems and their relation to lymph node metastasis. Nat J Community Med, 2, 136-42.

Erika R, Ingrid B, Masamichi B, et al (2004). Neoexpression of N-cadherin in E-cadherin positive colon cancers. Int $J$ Cancer, 111, 711-9.

Gaoliang O (2011). Epithelial-mesenchymal transition and cancer stem cells. Cancer stem cell-The cutting edge, Edited by Prof. Stanley Shostak, pp 167-88.

Kathryn R, Lawson MJW, Keith RJ (2006). Modulation of E- and $\mathrm{N}$-cadherin levels in oral squamous carcinoma cells reveals $\mathrm{N}$-cadherin-specific increases in invasion-related signaling pathways. Proc Amer Assoc Cancer Res, 47.

Ke L, Xin W, Wei H, et al (2009). Expression of N-cadherin in esophageal squamous cell carcinoma and silencing expression of N-cadherin using RNA interference on invasiveness of EC9706 cells. Chinese J Cancer, 28, 8-13.

Lara DMD, Mar CEB (2004). N-cadherin in the spotlight of cell-cell adhesion, differentiation, embryogenesis, invasion and signaling. Int J Dev Biol, 48, 463-76.

Margaret JW, Yasushi S, Masato M, et al (2007). Cadherin switching. J Cell Sci, 121, 727-35.

Marvin TN, Ryan S, Keith R, et al (1999). N-Cadherin promotes motility in human breast cancer cells regardless of their E-Cadherin expression. J Cell Biol, 147, 631-43.

Masato M, Keith RJ, Margaret JW (2004). Cadherin switching: Essential for behavioral but not morphological changes during an epithelium to mesenchyme transition. $J$ Cell Sci, 118, 873-87.

Mengzhu G, Yun M, Dahai Yu, et al (2018). Comparison of the expression of TGF- $\beta 1$, E-cadherin, N-cadherin, TP53, RB1CC1 and HIF-1 $\alpha$ in oral squamous cell carcinoma and lymph node metastases of humans and mice. Oncol Lett, 15, 1639-45.

MichaelZ, Eric GN(2009). Biomarkers for epithelial-mesenchymal transitions. J Clin Invest, 119, 1429-37.

Nakashima T, Huang C, Liu D, et al (2003). Neural -cadherin expression associated with angiogenesis in non-small-cell lung cancer patients. Br J Cancer, 88, 1727-33.

Nguyen PT, Kudo Y, Yoshida M, et al (2011). N-cadherin expression is involved in malignant behavior of head and neck cancer in relation to epithelial-mesenchymal transition. Histol Histopathol, 26, 147-56.

Nhan LT, Raymond BN, Anne EC, et al(1999). N-Cadherin expression in human prostate carcinoma cell lines. $\mathrm{Am} \mathrm{J}$ Pathol, 155, 787-98.

Rajendran R, Sivapathasundaram B (2012). Shafer's textbook of Oral Pathology.7th edition, pp 101-6.

Silvana P, Giuseppe P (2012). Epithelial-mesenchymal interactions in oral cancer metastasis. Oral cancer, Edited by Dr. Kalu U. E. Ogbureke.March, pp 373-88.

Sung WPYO, Mitsuyoshi H, Young SK, et al (2007). Expression of E-cadherin,P-cadherin and N-cadherin in oral squamous cell carcinoma: Correlation with the clinicopathologic features and patient outcome. J Craniomaxillofac Surg, 35, 1-9.

Suttichai K, Anak I (2012). Epithelial-mesenchymal transition in oral squamous cell carcinoma. SRN Oncol, 2012,1-10.

Yuu O-H, Sachiko S, Mutsunori F, et al (2017). Prognostic 
Harishchandra Rai K and Junaid Ahmed

prediction of oral squamous cell carcinoma by E-Cadherin and $\mathrm{N}$-Cadherin expression in overall cells in tumor nests or tumor cells at the invasive front. Cancer Microenvironment, 10, 87-94.

\section{(ब) $(\mathbb{8}$}

This work is licensed under a Creative Commons AttributionNon Commercial 4.0 International License. 